\title{
Design of off-axis PIAACMC mirrors
}

\author{
Eugene Pluzhnik ${ }^{a}$, Olivier Guyon ${ }^{b}$, Ruslan Belikov ${ }^{a}$, Brian Kern ${ }^{c}$, Eduardo Bendek ${ }^{a}$. \\ ${ }^{a}$ NASA Ames Research Center, Moffett Field, CA 94035, USA; \\ ${ }^{b}$ Steward Observatory, The University of Arizona, 933 N. Cherry Ave., Tucson, AZ 87521, \\ USA; \\ ${ }^{c}$ Jet Propulsion Laboratory, California Institute of Technology, 4800 Oak Grove Dr, Pasadena, \\ CA 91109, USA.
}

\begin{abstract}
The Phase-Induced Amplitude Apodization Complex Mask Coronagraph (PIAACMC) provides an efficient way to control diffraction propagation effects caused by the central obstruction/segmented mirrors of the telescope. PIAACMC can be optimized in a way that takes into account both chromatic diffraction effects caused by the telescope obstructed aperture and tip/tilt sensitivity of the coronagraph. As a result, unlike classic PIAA, the PIAACMC mirror shapes are often slightly asymmetric even for an on-axis configuration and require more care in calculating off-axis shapes when an off-axis configuration is preferred.

A method to design off-axis PIAA mirror shapes given an on-axis mirror design is presented. The algorithm is based on geometrical ray tracing and is able to calculate off-axis PIAA mirror shapes for an arbitrary geometry of the input and output beams. The method is demonstrated using the third generation PIAACMC design for WFIRST-AFTA telescope. Geometrical optics design issues related to the off-axis diffraction propagation effects are also discussed.
\end{abstract}

Keywords: Exoplanets, Coronagraphy, PIAA, Optics Design

\section{INTRODUCTION}

The WFIRST-AFTA coronagraph is designed to have powerful high contrast performance for direct imaging of exoplanets by their reflected light. Any coronagraph system considered for the WFIRST-AFTA project should solve the main design problem how to remove the star light diffracted from the telescope central obstruction. The

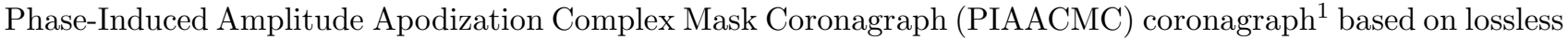
pupil apodization by the beam shaping concept ${ }^{2}$ (PIAA coronagraph) combined with a complex amplitude focal plane mask gives a solution of this problem.

The PIAACMC coronagraph can be optimized in a way that takes into account both chromatic diffraction effects caused by the telescope obstructed aperture, and tip/tilt sensitivity of the coronagraph, ${ }^{3}$ and still attain the performance required by AFTA. ${ }^{4}$ The typical design process for PIAA mirrors is to first optimize their shapes for an abstract (unphysical) on-axis collimated space configuration and then convert this design to a real unobstructed off-axis physical configuration. The conversion to off-axis is not trivial and the goal of this paper is to present a method to calculate PIAA optics for an arbitrary off-axis geometry of the optical system based on the optimized on-axis optics shapes. By "arbitrary", we mean different off-axis geometries for the system entrance and exit beams as well as a possible beam compression that the system should perform. Though diffraction critically affects the final system performance, our consideration will be based on geometrical optics only, assuming that the diffraction propagation effects are taken into account during the on-axis system optimization and are unaffected by conversion to off-axis. 


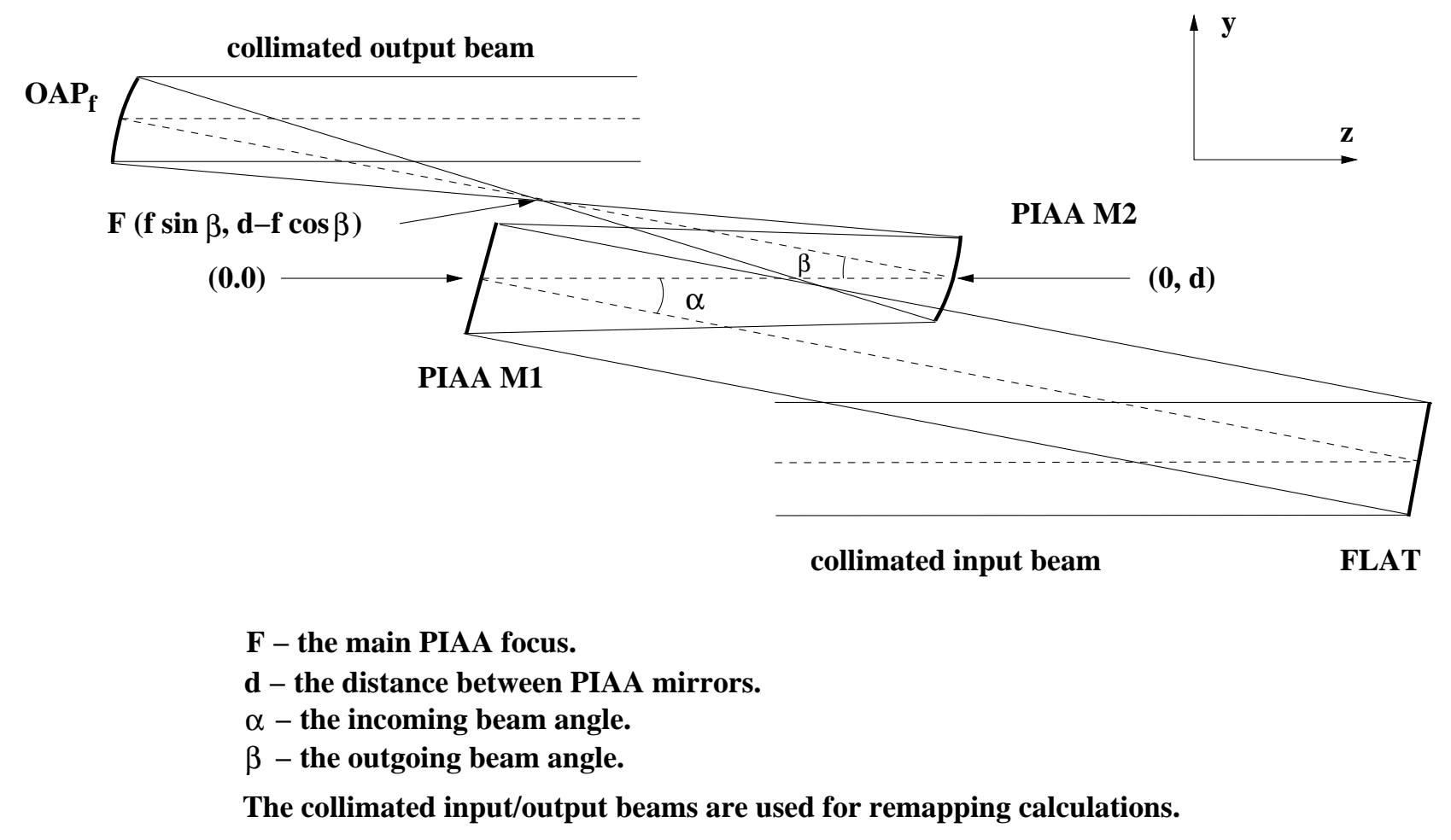

Figure 1. Optical layout.

\section{OPTICAL LAYOUT}

The proposed optical layout ${ }^{3}$ of the PIAA system for the WFIRST-AFTA PIAACMC coronagraph (Fig. 1) includes two PIAA mirrors M1 and M2. In this collimated beam-to focus system the collimated input beam is reshaped by the M1 mirror and is focused by the M2 mirror. The angle of the input beam $\alpha$ is equal to $20^{\circ}$ (approx.) and the output beam angle $\beta$ is equal to $25^{\circ}$ (approx.). The input beam diameter is about $40 \mathrm{~mm}$, the distance $d$ between mirrors is about $750 \mathrm{~mm}$, and the focal length $f$ of M2 (the distance between the M2 center and the system focus) is about $1200 \mathrm{~mm}$. The M1 mirror also performes the compression of the input beam needed to match the focal plane PSF size with the diameter of the focal plane occulter. As a result the beam diameter on the M2 is approximately equal to $15 \mathrm{~mm}$.

We define a coordinate reference frame shown in Fig. 1 with the origin at the center of PIAA M1 and the $z$ axis parallel to the central ray between PIAA M1 and M2. All mirror shapes will be expressed in this reference frame as functions of $(x, y)$.

The base shape of the M1 and M2 mirrors (i.e. without any PIAA remapping) are a plane and an off-axis parabola (OAP) respectively. All the following mirror shapes are calculated as corrections to these shapes that are given by:

$$
\begin{array}{r}
P \operatorname{LANE}(x, y)=y \times \tan (\alpha / 2), \\
O A P(x, y)=\frac{f^{2} \sin ^{2} \beta-x^{2}-(y-f \sin \beta)^{2}}{2 f(1+\cos \beta)}+d .
\end{array}
$$

The base shapes are computed such that they focus the collimated input beam to the desired system focus whose coordinates are $(x, y, z)=(0, f \sin \beta, d-f \cos \beta)$ as shown in Fig. 1. Such a choice of base shapes turns the

\footnotetext{
Further author information: (Send correspondence to Eugene Pluzhnik) Eugene Pluzhnik: E-mail: yevgeniy.a.pluzhnyk@nasa.gov, Telephone: 16506041241
} 


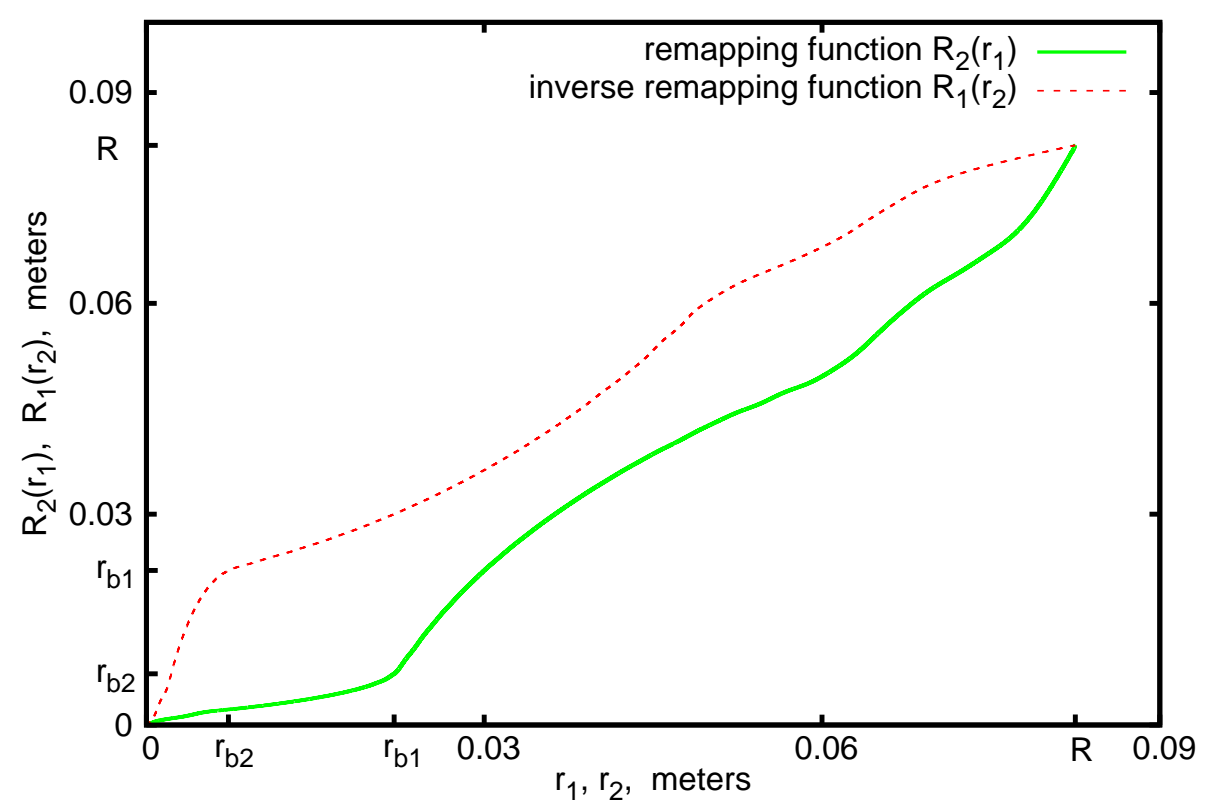

Figure 2. Extended remapping function $R_{2}\left(r_{1}\right)$ and inverse remapping function $R_{1}\left(r_{2}\right)$ : the working beam radii $r_{b 1}$ and $r_{b 2}$ are shown.

calculation of these shapes into a trivial procedure that is independent on a particular focus position and the beam compression/extending rate.

Two additional mirrors are used to perform remapping calculations. They are the flat mirror $F L A T$ and the off-axis parabola $O A P_{f}$ :

$$
\begin{array}{r}
F L A T(x, y)=\frac{2 d}{\cos \alpha}+\left(y+\frac{2 d}{\sin \alpha}\right) \tan (\alpha / 2), \\
O A P_{f}(x, y)=\frac{-f^{2} \sin ^{2} \beta+x^{2}+(y-f \sin \beta)^{2}}{2 f(1+\cos \beta)}+d-2 f \cos \beta .
\end{array}
$$

Both mirrors form collimated input and output beams that are parallel to the optical axis between PIAA M1 and M2 mirrors.

\section{OFF-AXIS MIRROR SHAPES}

In this section the procedure to calculate off axis PIAA shapes is given. The shape of both mirrors can be presented as a sum of three terms:

$$
\begin{array}{r}
M_{1}(x, y)=P L A N E(x, y)+f_{1}(r)+O A T_{1}(x, y) \\
M_{2}(x, y)=O A P(x, y)+f_{2}(r)+O A T_{2}(x, y) .
\end{array}
$$

In Eq. $3 P L A N E(x, y)$ and $O A P(x, y)$ are base shapes of PIAA M1 and M2 mirrors (Eq. 1), $f_{1}(r)$ and $f_{2}(r)$ are the circularly symmetric mirror terms that are mainly responsible for the beam remapping in the on-axis collimated beam-to-focus system, and $O A T_{1}(x, y)$ and $O A T_{2}(x, y)$ are the off-axis PIAA correction terms that preserve the correct PIAA apodization when mirrors are converted to off-axis. 


\subsection{Remapping functions}

The ray tracing calculations of the PIAA optics are based on the remapping function concept. For an on-axis source the remapping function establishes a correspondence between a ray position $\mathbf{r}_{\mathbf{1}}=\left(x_{1}, y_{1}\right)$ in the entrance pupil and the position $\mathbf{r}_{\mathbf{2}}=\left(x_{2}, y_{2}\right)$ of the same ray in the exit pupil:

$$
\begin{aligned}
& x_{2}=x_{2}\left(x_{1}, y_{1}\right) \\
& y_{2}=y_{2}\left(x_{1}, y_{1}\right) .
\end{aligned}
$$

Note, that the origin of the reference frames in Eq. 4 matches with the position of the central ray in the entrance and exit pupils.

Since we take the on-axis mirror shapes as given, the ray positions in the entrance and exit pupils, and thus 2D remapping functions $x_{2}\left(x_{1}, y_{1}\right)$ and $y_{2}\left(x_{1}, y_{1}\right)$, can be readily calculated with a ray tracing procedure.

The starting point to design mirror shapes for the WFIRST-AFTA coronagraph was the diffraction-based optimized solution obtained for an on-axis collimated beam to collimated beam PIAA system without beam compression. ${ }^{3}$ Although this solution is not circularly symmetric because of the central obstruction asymmetry, the deviation from the symmetry for both mirrors is small. That makes it possible to define the azimuthally averaged remapping function

$$
r_{2}\left(r_{1}\right)=<\sqrt{x_{2}^{2}\left(r_{1} \cos \phi, r_{1} \sin \phi\right)+y_{2}^{2}\left(r_{1} \cos \phi, r_{1} \sin \phi\right)}>_{\phi}
$$

that determines the radial mirror terms $f_{1}(r)$ and $f_{2}(r)$. In Eq. $5<\ldots>_{\phi}$ means azimuthal averaging.

The remapping function $r_{2}\left(r_{1}\right)$ constructed in according with Eq. 5 provides a good initial approximation to calculate the off-axis mirror shapes for all the rays within the working beam. However, the discontinuity of derivatives of the remapping function at the beam edge and an absence of the remapped values outside of the working beam create numerous difficulties for the ray tracing calculations that suppose to work with real square arrays of points.

To avoid these problems we created an extended remapping function $R_{2}\left(r_{1}\right)$ (Fig. 2) by transposing the remapping function $r_{2}\left(r_{1}\right)$ onto the intervals $\left[r b_{1}, R\right]$ and $\left[r b_{2}, R\right]$ backwards, beyond the working beam radii $r_{b 1}$ (on the PIAA M1) and $r_{b 2}$ (on the PIAA M2), and making sure that the derivatives match on the beam edge. The $R_{2}\left(r_{1}\right)$ is given as follows:

$$
\begin{gathered}
R_{2}\left(r_{1}\right)=r_{2}\left(r_{1}\right) \\
\left\{\begin{array}{l}
r_{1}=r_{b 1}+\left(1+\alpha_{1} r_{1}^{\prime}\right) r_{1}^{\prime} \\
r_{2}=r_{b 2}+\left(1+\alpha_{2} r_{2}^{\prime}\right) r_{2}^{\prime} \\
r_{b 2}-r_{2}^{\prime}=R_{2}\left(r_{b 1}-r_{1}^{\prime}\right)
\end{array} \quad \text { for } \quad r_{b 1} \leq r_{1} \leq R,\right.
\end{gathered}
$$

where

$$
\begin{aligned}
\alpha_{1} & =\frac{\left(R-r_{b 1}\right) / r_{b 1}-1}{r_{b 1}}, \\
\alpha_{2} & =\frac{\left(R-r_{b 2}\right) / r_{b 2}-1}{r_{b 2}},
\end{aligned}
$$

and $R$ is the radius of an area big enough to cover all arrays involved in the mirror shapes calculation.

It should be noted that for a system with the beam compression $r_{b 2}$ is not equal to $r_{b 1}$. The desired beam compression can be also included in the final expressions for $2 \mathrm{D}$ remapping functions $X_{2}\left(x_{1}, y_{1}\right)$ and $Y_{2}\left(x_{1}, y_{1}\right)$ through the rescaling factor $r_{b 2} / r_{b 1}$ :

$$
\begin{aligned}
& X_{2}\left(x_{1}, y_{1}\right)=\frac{r_{b 2}}{r_{b 1}} x_{2}\left(x_{1}, y_{1}\right) \\
& Y_{2}\left(x_{1}, y_{1}\right)=\frac{r_{b 2}}{r_{b 1}} y_{2}\left(x_{1}, y_{1}\right),
\end{aligned}
$$

where $x_{2}\left(x_{1}, y_{1}\right)$ and $y_{2}\left(x_{1}, y_{1}\right)$ are remapping functions for the system without the beam compression. 


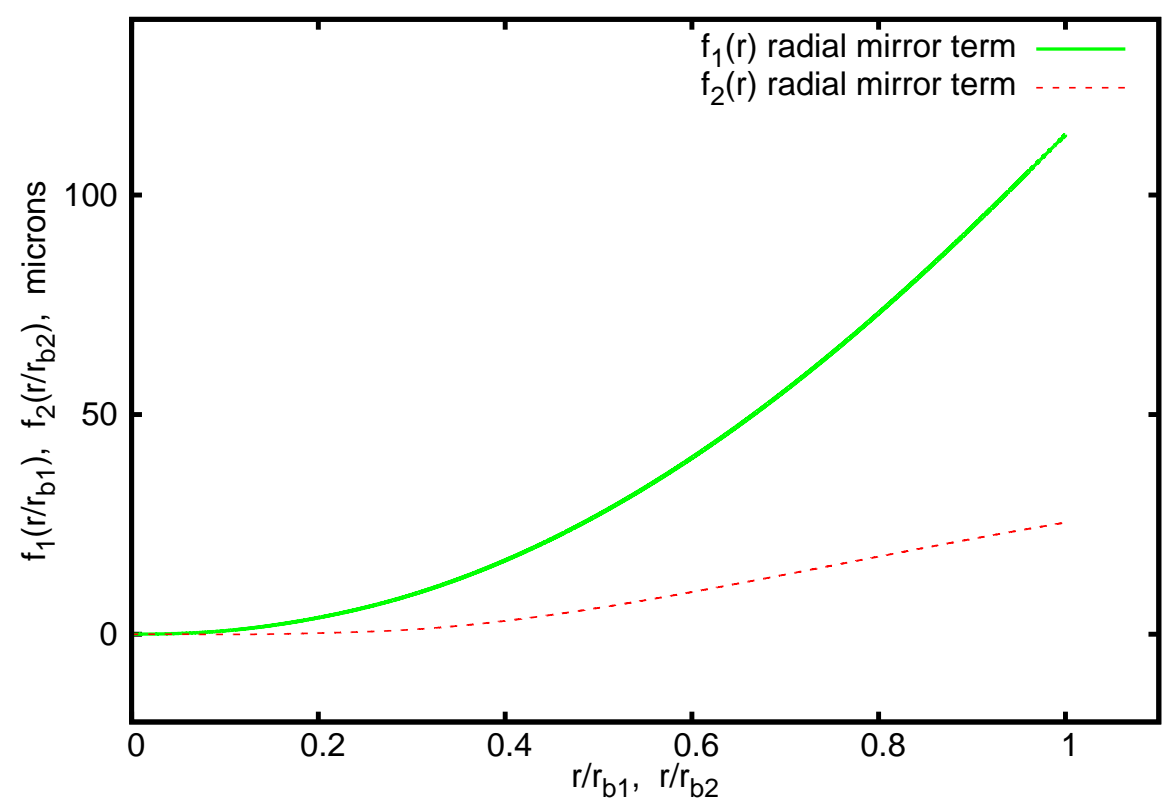

Figure 3. Mirror shapes for WFIRST-AFTA coronagraph: radial mirror terms, the focal distance $f$ is equal to the distance between mirrors $d$.

\subsection{Optics shapes calculation}

The following iteration procedure is proposed to calculate off-axis PIAA mirror shapes given on-axis ones:

1. Calculate the 2-D remapping functions $x_{2}\left(x_{1}, y_{1}\right)$ and $y_{2}\left(x_{1}, y_{1}\right)$ by ray tracing on-axis mirror shapes and azimuthally average them to get the radial remapping function $R_{2}\left(r_{1}\right)$.

2. Calculate the 2-D remapping functions $X_{2}\left(x_{1}, y_{1}\right)$ and $Y_{2}\left(x_{1}, y_{1}\right)$ for the compressed beam.

3. Determine base shapes $P L A N E(x, y)$ and $O A P(x, y)$ of mirrors per Eq. 1.

4. Calculate radial mirror profiles $f_{1}(r)$ and $f_{2}(r)$ for on-axis collimated beam-to-focus system from $R_{2}\left(r_{1}\right)$ (Section 3.3).

5. Start a loop. For i-th iteration step construct an estimate $O A T_{1, i}(x, y)$ as sum of small correcting terms $d M_{1, j}(x, y)$ calculated during previous iteration steps (Section 3.4 ):

$$
\begin{aligned}
& O A T_{1, i}(x, y)=\sum_{j=0}^{i} d M_{1, j}(x, y), \\
& O A T_{1,0}(x, y)=0 .
\end{aligned}
$$

6. Determine off-axis PIAA term $O A T_{2, i}(x, y)$ that provides equal optical path length (OPL) for all the rays propagated from the source to the main PIAA focus system. This step is performed assuming the off-axis PIAA term $O A T_{1}(x, y)$ is equal to $O A T_{1, i}(x, y)$.

7. Close the loop by returning to step 5 . 


\subsection{Radial mirror terms}

For the case of a radially symmetric collimated-beam to collimated-beam PIAA system, the mirror shapes can be directly computed from $R_{2}\left(r_{1}\right)$ by numerically integrating a pair of differential equations. ${ }^{2}$

In this section we consider the case of the on-axis collimated-beam-to-focus radially symmetric system. The proposed solution is similar to the procedure we use to calculate $2 \mathrm{D}$ off-axis later in this paper.

The radial mirror profiles $f_{1}(r)$ can be derived from

$$
f_{1}(r)=f_{1}(0)+\int_{0}^{r} \frac{d f_{1}(t)}{d t} d t
$$

if the derivative $d f_{1}(r) / d r$ is known. The derivative $d f_{1}(r) / d r$ can be easily estimated for a collimated beam-tofocus system by using the "desired" normal (i.e. one that generates the desired remapping in according with Eq. 5) to the M1 surface.

In according with the reflection law the "desired" normal $\mathbf{n}_{\mathbf{i}}=\left(n_{r}^{i}, n_{z}^{i}\right)$ to the surface of the M1 mirror can be written as

$$
\mathbf{n}_{\mathbf{i}}\left(\mathbf{r}_{\mathbf{1}}\right)=\left(n_{r}^{i}, n_{z}^{i}\right)=\frac{\mathbf{r}_{\mathbf{1 s}}}{r_{1 s}}+\frac{\mathbf{r}_{\mathbf{1 2}}}{r_{12}},
$$

where $\mathbf{r}_{1 \mathbf{s}}$ is the vector from a point $\mathbf{r}_{\mathbf{1}}$ on the M1 surface to the source, and the vector $\mathbf{r}_{\mathbf{1 2}}$ connects point $\mathbf{r}_{\mathbf{1}}$ with the remapped point $\mathbf{r}_{\mathbf{2}}$. The position of the remapped point $\mathbf{r}_{\mathbf{2}}$ on the M2 mirror is determined by the remapping function $R_{2}\left(r_{1}\right)$. The derivative $d f_{1}(r) / d r$ is given by

$$
\frac{d f_{1}(r)}{d r}=-\frac{n_{r}^{i}}{n_{z}^{i}}
$$

To derive both $f_{1}(r)$ and $f_{2}(r)$ radial profiles an iterative approach is used. In this approach, the "desired" normal $\mathbf{n}_{\mathbf{i}}$ is estimated from the previous approximation for the M1 and M2 shapes and the M1 shape is obtained by solving Eq. 9. The next approximation of the M2 shape is calculated assuming that for all the rays propagated from the source to the main PIAA focus OPL should be equal to the OPL of the principal ray (Fermat's principle). The OPL of the principal ray is known from the initial conditions. Radial mirror terms for the WFIRST-AFTA coronagraph are shown in Fig. 3.

\subsection{Calculation of $d M_{1}(x, y)$}

To calculate the corrective term $d M_{1, j}(x, y)$ (Section 3.2) we should:

1. For given $2 \mathrm{D}$ remapping functions (Eq. 7) determine the "desired" normal $\mathbf{n}_{\mathbf{i}}=\left(n_{x}^{i}, n_{y}^{i}, n_{z}^{i}\right)$ to the M1 surface assuming that M1 and M2 mirror shapes match the current shapes approximation. Similarly Eq. 10, the "desired" normal

$$
\mathbf{n}_{\mathbf{i}}(\mathbf{x}, \mathbf{y})=\left(n_{x}^{i}, n_{y}^{i}, n_{z}^{i}\right)=\frac{\mathbf{r}_{1 \mathbf{s}}}{r_{1 s}}+\frac{\mathbf{r}_{\mathbf{1 2}}}{r_{12}}
$$

where vectors $\mathbf{r}_{1 \mathbf{s}}$ and $\mathbf{r}_{12}$ are determined in Section 3.3. To determine a particular ray position on the M2 surface the geometrical propagation in direction from the exit pupil to the off-axis parabola $O A P_{f}$ and then to the M2 mirror should be used. The ray position in the exit pupil can be determined as the remapped (in accordance with Eq. 7) ray position in the entrance pupil. By the entrance pupil we mean a plane upstream of the flat mirror $F L A T(x, y)$ (Eq. 2). The exit pupil is the plane downstream of the off-axis parabola $O A P_{f}(x, y)$ (Eq. 2) optically conjugated with the M2 mirror. Both the entrance and exit pupils are orthogonal to the collimated input/output beams and all the rays are considered to be collimated between the entrance pupil and the flat mirror and between the exit pupil and $O A P_{f}$.

2. Obtain the "current" normal (i.e. gradient vector of the surface) $\mathbf{n}_{\mathbf{c}}=\left(n_{x}^{c}, n_{y}^{c}, n_{z}^{c}\right)$ to the current M1 mirror shape. 

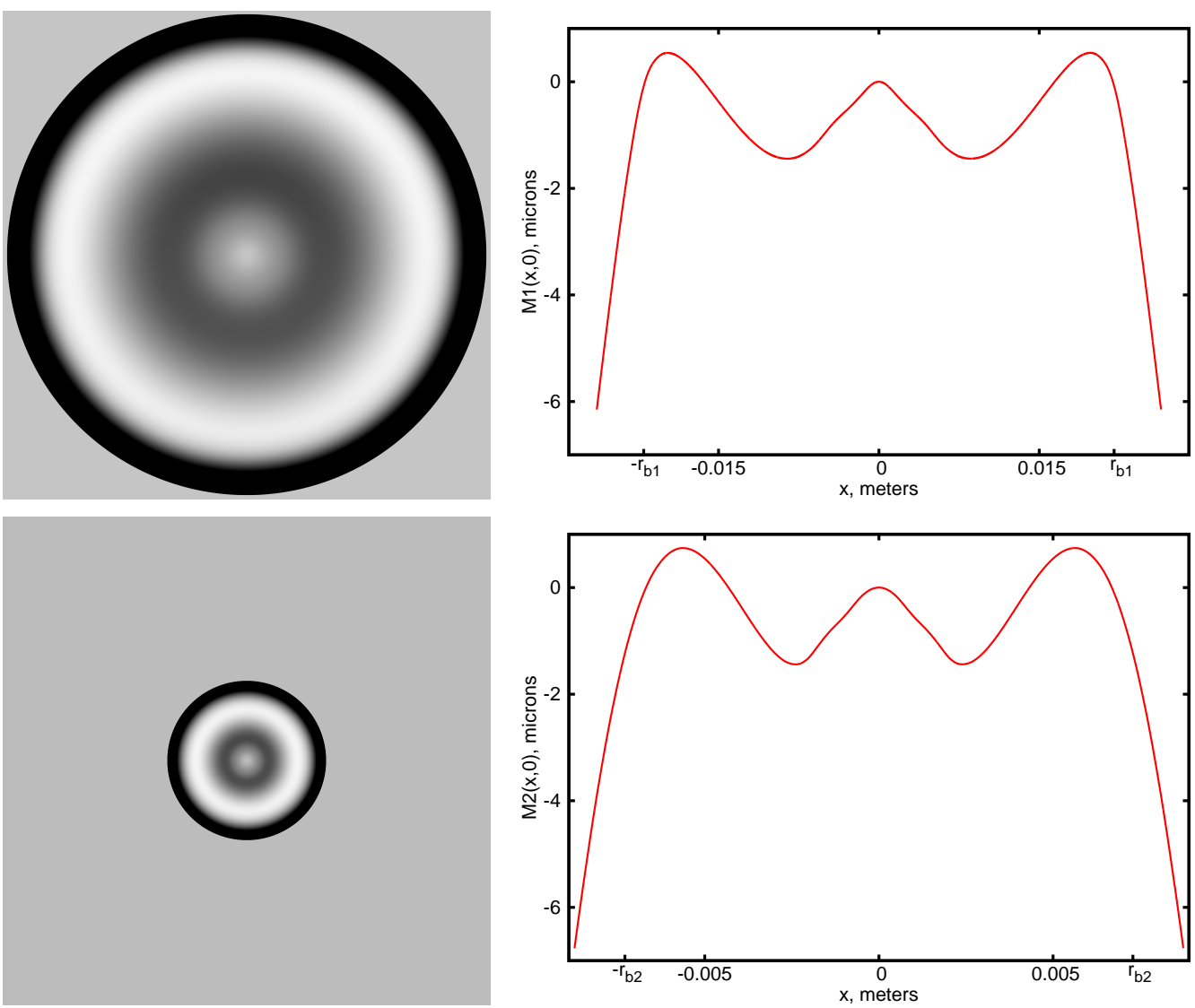

Figure 4. PIAA M1 (top) and M2 (bottom) off-axis shapes for the WFIRST-AFTA coronagraph.

Working beam radii $r_{b 1}$ and $r_{b 2}$ are shown on crosscuts on the right.

3. Calculate derivatives $\partial d M_{1, i}(x, y) / \partial x$ and $\partial d M_{1, i}(x, y) / \partial y$ :

$$
\begin{gathered}
\partial d M_{1, i}(x, y) / \partial x=-n_{x}^{i} / n_{z}^{i}+n_{x}^{c} / n_{z}^{c}, \\
\partial d M_{1, i}(x, y) / \partial y=-n_{y}^{i} / n_{z}^{i}+n_{y}^{c} / n_{z}^{c} .
\end{gathered}
$$

4. Calculate the $d M_{1, i}(x, y)$ through a line integral

$$
d M_{1, i}(x, y)=\int_{(0,0)}^{(x, y)} \frac{\partial d M_{1, i}(x, y)}{\partial x} d x+\frac{\partial d M_{1, i}(x, y)}{\partial y} d y
$$

Since, for any physically possible remapping the integration in Eq. 15 along any path that connects the mirror center with the point $(x, y)$ should give the same result, $d M_{1, i}(x, y)$ values can be also averaged for different integration paths.

5. To reduce the M1 errors caused by the numerical integration/interpolation an appropriate smoothing algorithm should be applied for each $d M_{1, i}(x, y)$ estimation.

\subsection{Calculation of $O A T_{2}(x, y)$}

To determine the $O A T_{2, i}(x, y)$ we use an iterative procedure II that corrects the OPL of the output rays on every step of the main iteration. Note that iteration procedure II is different from the main iteration discussed earlier in Sec. 3.2. 

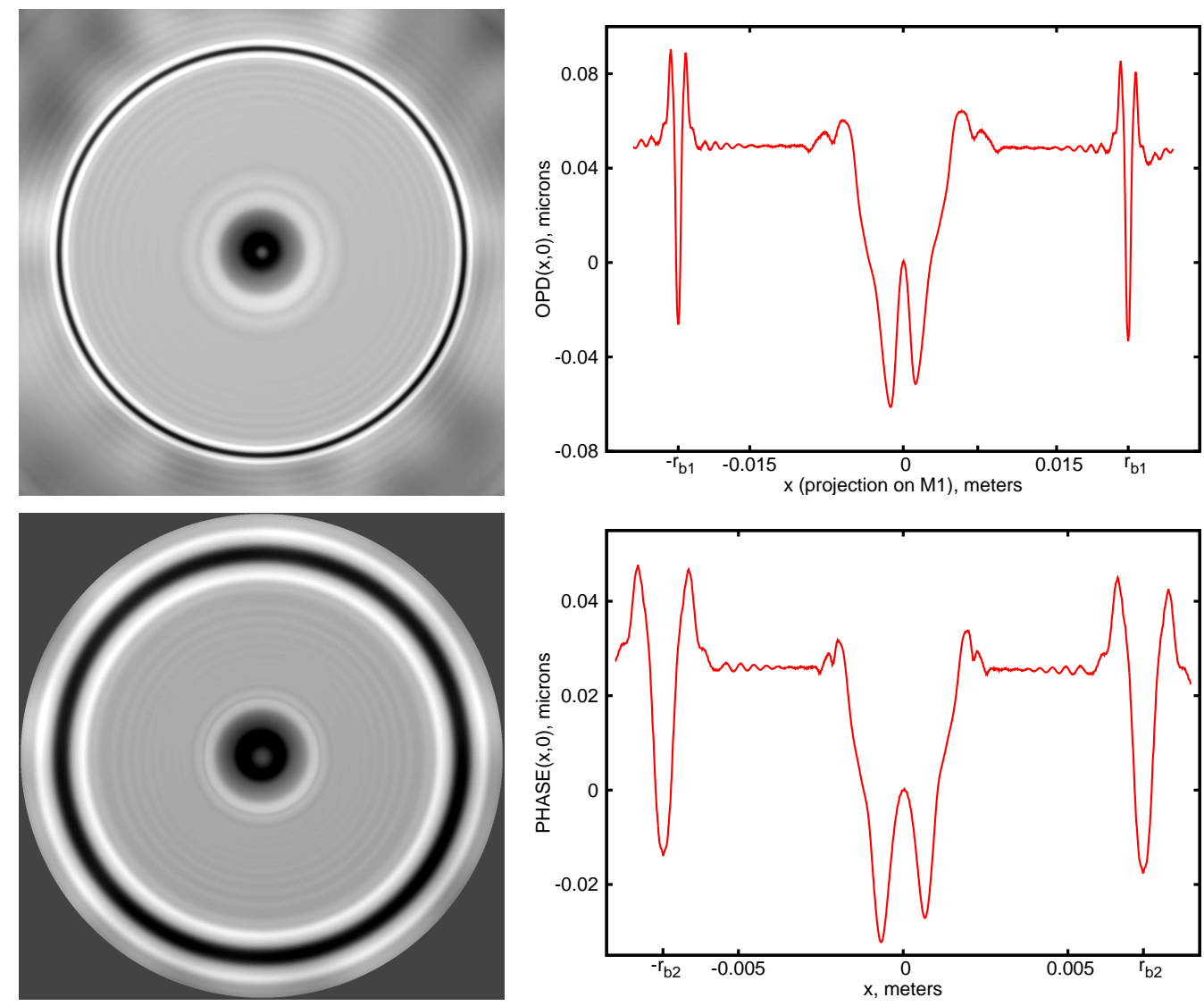

Figure 5. The output beam geometrical $\operatorname{OPD}(\mathrm{x}, \mathrm{y})$ for the on-axis optimized shapes in projection on the M1 (top) and the PHASE(x,y) mirror term (bottom). Note that $O P D(x, y)$ and $P H A S E(x, y)$ have different scales.

During k-th step of the iteration II the function $d M_{2, k}\left(x_{2}, y_{2}\right)$ is calculated:

$$
d M_{2, k}\left(x_{2}, y_{2}\right)=-\frac{O P L_{k}\left(x_{2}, y_{2}\right)-O P L(0,0)}{2},
$$

where $O P L_{k}(x, y)$ is the optical path length of the ray that starts at the source position, reflects from the current approximation of the M1 shape at the point $\left(x_{1}, y_{1}\right)$, reflects from the current approximation M2 shape at the point $\left(x_{2}, y_{2}\right)$ and then hits the system focus. Similary to $O A T_{1, i}(x, y)$, the off-axis term $O A T_{2, i}(x, y)$ can be determined as

$$
\begin{aligned}
O A T_{2, i}\left(x_{2}, y_{2}\right) & =\sum_{k} d M_{2, k}\left(x_{2}, y_{2}\right), \\
O A T_{2,0}\left(x_{2}, y_{2}\right) & =0 .
\end{aligned}
$$

The iteration stops as soon as the OPD for all the rays that cross the M2 surface becomes negligible. Because of the remapping, the sampling of $O A T_{2}\left(x_{2}, y_{2}\right)$ on the M2 surface appears to be inhomogenious, even if the original arrays sampling on the M1 surface is homogenious. To get homogeneous $O A T_{2}\left(x_{2}, y_{2}\right)$ sampling on the M2 surface a triangulation procedure should be used.

\section{MIRROR SHAPES}

The calculated mirror shapes for the WFIRST-AFTA coronagraph are shown in Fig. 4. To present the shapes deviation from common optical shapes, the parabolic terms given by

$$
P_{1}(x, y)=0.171215209 \times y+0.24227703 \times\left(x^{2}+y^{2}\right), \quad \text { subtructed from PIAA M1 }
$$




$$
P_{2}(x, y)=0.228083501 \times y+0.64898643 \times\left(x^{2}+y^{2}\right) \quad \text { subtructed from PIAA M2. }
$$

were pre-subtracted from the mirror surfaces. Note that these parabolas are not the base optics shapes considered earlier and are not chosen based on their optical properties, they were chosen simply to highlight higher order departures visually.

An additional point to emphasize is that the diffraction propagation introduces radial oscillations both in amplitude and in phase of the output beam (Fig. 5,6) which the initially optimized on-axis mirror shapes take into account and correct for.

As a result the geometrical OPD of the output beam is not equal to zero according to a purely geometrical optics model (Fig. 5), although it appears to be equal to zero if we account for diffraction propagation. To take into account the above effect an additional term for the M2 mirror shape PHASE $(x, y)$ should be introduced:

$$
M_{2}(x, y)=O A P(x, y)+f_{2}(r)+O A T_{2}(x, y)+P H A S E(x, y),
$$

where $O_{A}(x, y)$ term describes the M2 shape assuming that the wavefront after M2 mirror is spherical, and $\operatorname{PHASE}(\mathrm{x}, \mathrm{y})$ takes into account the OPD change caused by the diffraction propagation in the system. To calculate the M2 shape in this case the equation Eq. 16 should be modified in the following way

$$
d M_{2, k}\left(x_{2}, y_{2}\right)=-\frac{O P L_{k}\left(x_{2}, y_{2}\right)-O P L(0,0)-O P D_{d}(x, y)}{2},
$$

where $O P D_{d}(x, y)$ is the geometrical OPD in the output beam for the baseline on-axis system.

The second problem related to the diffraction caused oscillations in the mirror shapes is the dramatic slow down of the procedure to calculate M2 shape when the residual OPD of the output beam reaches $10^{-10} \mathrm{~m}$ level.

\section{RAY TRACING CHECK}

The derived optical shapes with the "phase" term $\operatorname{PHASE}(x, y)=0$ have been checked with the ray tracing code that has been developed to simulate the previous generations of PIAA optics manufactured by AXSYS Inc. ${ }^{5}$ and Tinsley Inc. ${ }^{6}$ Results of the ray tracing simulation shown in Fig. 6

The complex amplitude in the exit pupil of the PIAA has been obtained by analysing the distribution of the $2 \times 10^{8}$ random rays is enough to estimate the amplitude profile with rms of residuals of about 0.09 . The derived amplitude profile clearly shows the radial amplitude oscillations. These oscillations would be corrected if the diffraction propagation is considered.

The phase deviations in the exit pupil are only limited by the residual OPD of the output beam caused by the convergence of the M2 calculation algorithm discussed above. Their rms reaches the $\lambda / 3000$ level that is comparable with the physically achievable limit for the optical quality.

\section{SUMMARY}

In this paper we presented a method to calculate off-axis mirror shapes for PIAA coronagraph assuming that the on-axis mirror shapes are known. The method is based on geometrical ray tracing and is able to calculate off-axis PIAA mirror shapes for an arbitrary geometry of the input and output beams.

\section{REFERENCES}

[1] Guyon, O., Martinache, F., Belikov, R., and Soummer, R., "High performance PIAA coronagraphy with complex amplitude focal plane masks," Astroph. J. Suppl. 190, 220-232 (2010).

[2] Guyon, O., "Phase-induced amplitude apodization of telescope pupils for extrasolar terrestrial planet imaging," Astron. and Astroph. 404, 379-387 (2003).

[3] Guyon, O., "High performance PIAACMC designs for centrally obscured and segmented apertures," in preparation. 
Exit pupil amplitude

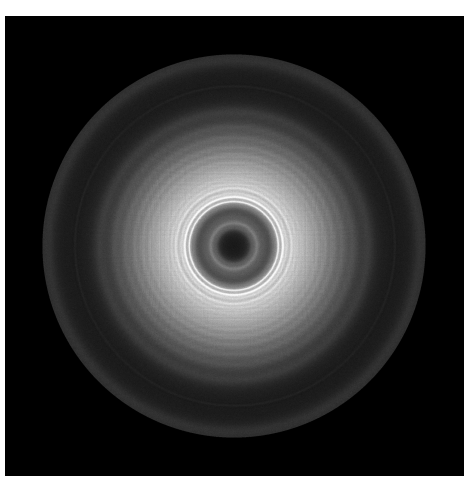

Complex amplitude in the remapped pupil

(on axis source, crosscut through the pupil center)

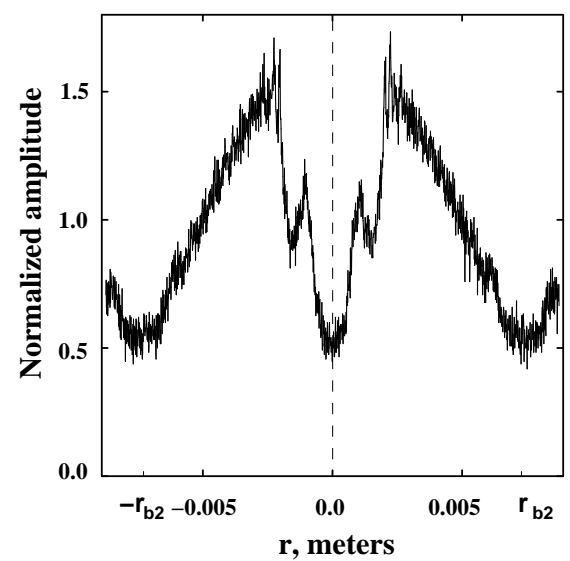

Figure 6. Three dimensional ray tracing check of the PIAA mirror shapes with the term $P H A S E(x, y)=0$ :

a) the exit pupil amplitude and phase;

b) the amplitude and phase crosscuts through the exit pupil center.

[4] Krist, J., "Numerical modelling of the proposed WFIRST-AFTA coronagraphs and their predicted performances," J. Astron. Tel. Instr. Syst., this issue.

[5] Guyon, O., Pluzhnik, E., Martinache, F., Totems, J., Tanaka, S., and Matsuo, T., "High-contrast imaging and wavefront control with a piaa coronagraph: Laboratory system validation," Publ. Astron. Soc. Pacif. 122, 71-84 (2010).

[6] Belikov, R., Pluzhnik, E., Witteborn, F., Greene, T., Lynch, D., Zell, P., and Guyon, O., "Laboratory demonstration of high-contrast imaging at inner working angles $2 \lambda / \mathrm{d}$ and better," Proc. SPIE 8151, 815102 (2011). 